\title{
Design and performance of a compact and stationary microSPECT system
}

\author{
Roel Van Holen, ${ }^{*}$ Bert Vandeghinste, Karel Deprez, and Stefaan Vandenberghe \\ ELIS Department, MEDISIP, Ghent University, iMinds, \\ De Pintelaan 185 block B, B-9000 Ghent, Belgium
}

(Dated: September 4, 2013) 


\begin{abstract}
Purpose: Over the past ten years, there has been an extensive growth in the development of microSPECT imagers. Most of the systems are based on the combination of conventional, relatively large gamma cameras with poor intrinsic spatial resolution and multi-pinhole collimators working in large magnification mode. Spatial resolutions range from 0.58 to $0.76 \mathrm{~mm}$ while peak sensitivities vary from $0.06 \%$ to $0.4 \%$. While pushing the limits of performance is of major importance, we believe that there is a need for smaller and less complex systems that bring along a reduced cost. While low footprint and low-cost systems can make microSPECT available to more researchers, the ease of operation and calibration and low maintainance cost are additional factors that can facilitate the use of microSPECT in molecular imaging. In this paper, we simulate the performance of a microSPECT imager that combines high space-bandwidth detectors and pinholes with truncated projection, resulting in a small and stationary system.

Methods: A system optimization algorithm is used to determine the optimal SPECT systems, given our high resolutions detectors and a fixed field-of-view. These optimal system geometries are then used to simulate of a Defrise disk phantom, a hot rod phantom. Finally, a MOBY mouse phantom, with realistic concentrations of Tc99m-tetrofosmin is simulated.

Results: Results show that we can successfully reconstruct a Defrise disk phantom of $24 \mathrm{~mm}$ in diameter without any rotating system components or translation of the object. Reconstructed spatial resolution is approximately $800 \mu \mathrm{m}$ while the peak sensitivity $0.23 \%$. Finally, the simulation of the MOBY mouse phantom shows that we can accurately reconstruct mouse images.

Conclusions: These results show that pinholes with truncated projections can be used in small magnification or minification mode to obtain a compact and stationary microSPECT system. We showed that we can reach state-of-the-art system performance and that we can successfully reconstruct images with realistic noise levels in a pre-clinical context. Such a system can be useful for dynamic SPECT imaging.
\end{abstract}

\footnotetext{
*Roel.VanHolen@UGent.be
} 


\section{INTRODUCTION}

Small animal Single Photon Emission Computed Tomography (microSPECT) has been recognized as one of the major in vivo molecular imaging techniques [e.g. 1]. It was soon accepted that multi-pinhole collimators provide the best trade-off between spatial resolution low-cost systems can make microSPECT available to more researchers, the ease of operation, 


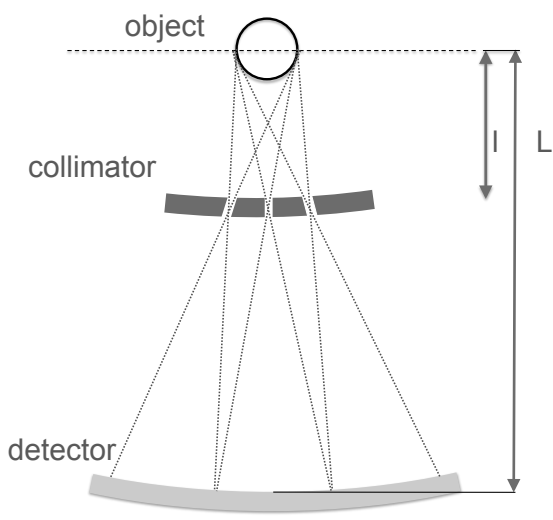

(a)

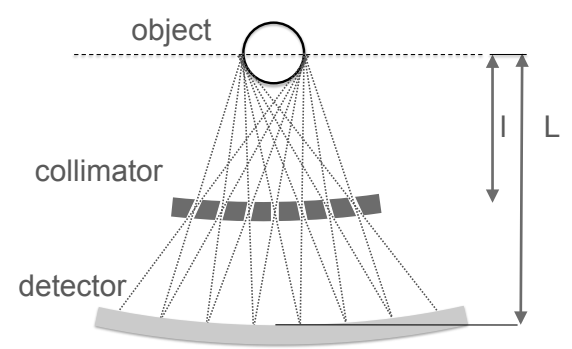

(b)

FIG. 1. Two-dimensional representation of a multipinhole collimator. (a) Traditionally, a pinhole magnifies an object onto the detector. This is achieved by making the detector-to-pinhole distance $(L-l)$ larger than the object-to-pinhole distance $l$. In (b), $(L-l)$ is smaller than $l$ and the source is minified onto the detector.

calibration and low maintainance cost are additional factors that can facilitate the use of microSPECT in molecular imaging. To make smaller systems with uncompromised system performance, we refer to a publication by Rogulski et al. that indicated that in pinhole-based SPECT, the trade-off between spatial resolution and sensitivity can be overcome using high space-bandwidth detectors [15]. The basic insight that leads to this theory is that with improvements in intrinsic detector spatial resolution, the detectors can be placed (without overlap) closer to the pinholes (figure 1). This in turn can lead to minified object projections onto the detector (figure 1 (b)). This means that smaller detectors can be used and as a consequence, more pinhole-detector pairs can be placed in the same space. On a system level, this can finally result in better sensitivity for equal spatial resolution [16]. In our lab, we are constructing a prototype system that exploits this principle, not to improve system performance, but to enable small, low cost and stationary microSPECT imaging at uncompromised performance.

In this paper, we propose a mouse/rat-brain multi-pinhole system based on compact, inexpensive, high-resolution detectors. The design combines these high space-bandwidth detectors with pinholes with truncated projections (pinholes that only partially see the field-of- 
view). First, we define the design constraints that partly originate from the physical size of the detector. Then, we optimize the system for point source sensitivity. Once the optimal design parameters are defined, we simulate different quality control phantoms: a Defrise disk phantom and a hot rod phantom. Finally, a whole body MOBY mouse phantom will be simulated, assuming a realistic Tc-99m-tetrofosmin tracer distribution.

\section{MATERIALS AND METHODS}

\section{A. Design constraints}

\section{Field-of-view, bore size and system resolution}

Since our system aims at rat brain and whole body mouse and imaging, the first design constraint concerns the size of the field-of-view (FOV). A typical FOV that is used for mouse and rat brain imaging is around $30 \mathrm{~mm}$ in transverse orientation. In our design, every individual pinhole measures a different transverse FOV of $12 \mathrm{~mm}$ diameter while all pinholes look at the same axial portion of $12 \mathrm{~mm}$ length. These values are based on literature values of a state-of-the-art system [17]. In order to cover the entire transverse section of the animal, we will not scan different bed positions [17], but rather employ the technique of truncated projections $[10,18]$. A second constraint requires a central bore of at least $40 \mathrm{~mm}$ diameter to allow sufficient space to accommodate a whole-body mouse or a rat head. This implies that the pinholes can be placed at a minimum distance $l$ of $20 \mathrm{~mm}$ from the center. Finally, we decide to target an analytic system resolution $R_{t}$ of $1.4 \mathrm{~mm}$, which is based on the general purpose mouse imaging protocol provided by three microSPECT vendors. Using this protocol, we recently showed that reconstructed resolutions from 0.58 to $0.76 \mathrm{~mm}$ can be obtained [14]. These discrepancy between reconstructed and target resolution can be explained by the use of resolution recovery in the iterative reconstruction algorithms. A flowchart of system optimization given a set of constraints can be found in figure 3 .

\section{Detector size and intrinsic resolution}

The design of our system is based on a previously developed high-resolution scintillation 


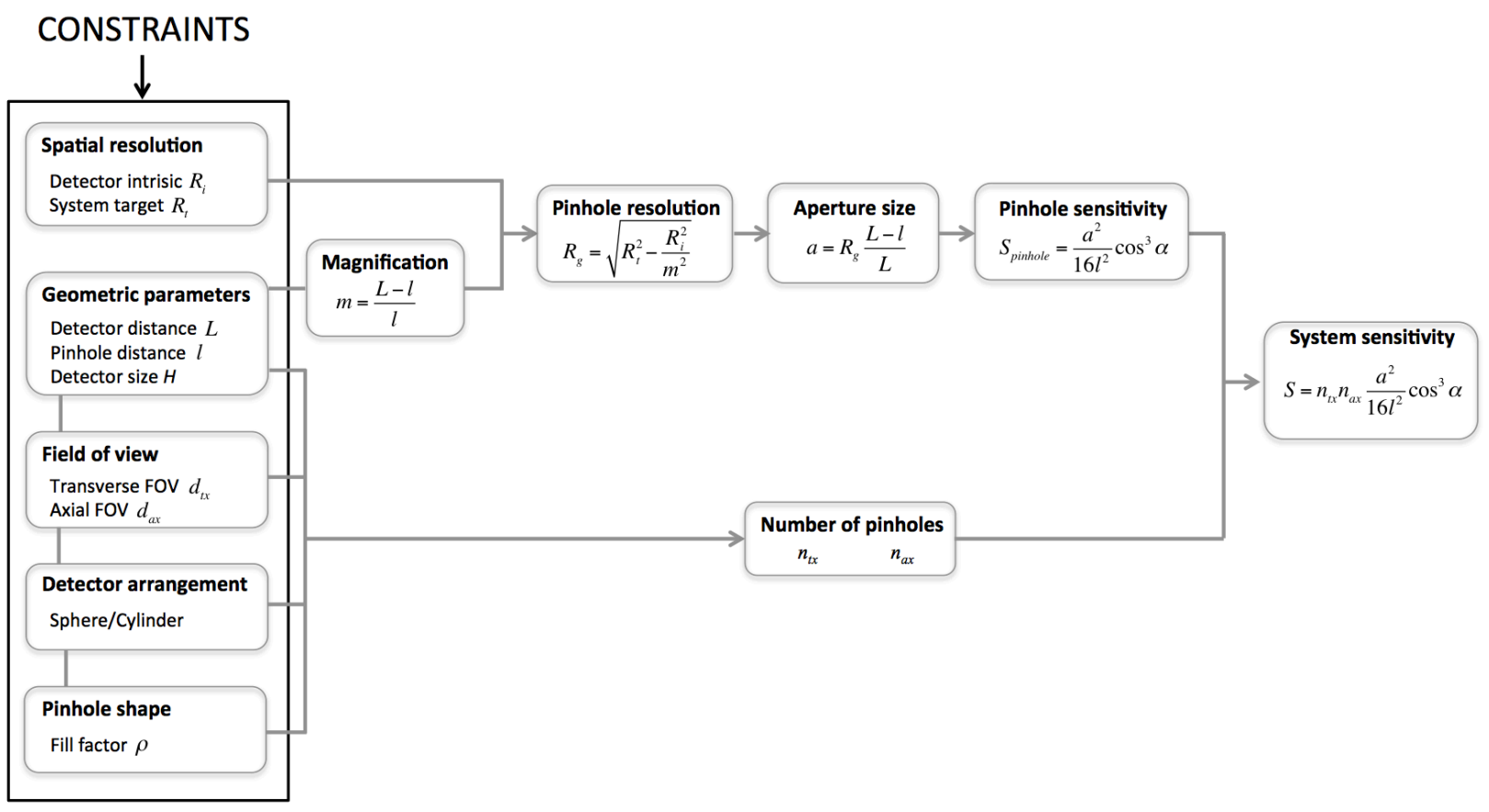

FIG. 2. System optimization procedure: From the geometric and other constraints, we calculate (i) the pinhole spatial resolution (ii) the number of pinholes that can be physically positioned without overlap on the detector. If we then write the single pinhole sensitivity as a function of pinhole spatial resolution, and multiply with the number of pinholes, we arrive at the full system sensitivity as a function of our constraints (we use a cylindrical detector arrangement).

H-8500 position sensitive PMT and a $49 \times 49 \times 5 \mathrm{~mm}^{3} \mathrm{NaI}(\mathrm{Tl})$ scintillator. This PMT has a grid of eight by eight $6 \times 6 \mathrm{~mm}^{2}$ anodes. Traditional PMT pre-amplifiers and pulse shapers feed all 64 anode signals to analog to digital converters. The digital signals are integrated in an FPGA and the outcome of the separate channels is sent to a host PC via Gigabit ethernet. The availability of the data of all individual channels allows for event positioning based on a statistical description of the detection of light photons [21]. Currently, we are able to achieve $800 \mu \mathrm{m}$ intrinsic resolution using Maximum Likelihood Position Estimation (MLPE), implemented as described by Hesterman et al. [22]. The use of this modular detector constrains the intrinsic resolution at $800 \mu \mathrm{m}$. Furthermore, the axial and transaxial dimensions of the system will also be determined by the size of the detector. Axially, we decide to use a single ring of detectors, which puts the axial detector size $H$ at $49 \mathrm{~mm}$. In practice, we will approximate the circular transaxial cross-section by 
TABLE I. Fixed system parameters as input for optimization

\begin{tabular}{|cc|l|}
\hline \hline Single pinhole transverse FOV & $d_{t x}$ & $12 \mathrm{~mm}$ \\
\hline Single pinhole Axial FOV & $d_{a x}$ & $12 \mathrm{~mm}$ \\
\hline Total axial FOV & $d_{\text {total }}$ & $30 \mathrm{~mm}$ \\
\hline Minimum Pinhole Distance & $l_{\text {min }}$ & $20 \mathrm{~mm}$ \\
\hline Total System Resolution & $R_{t}$ & $1.4 \mathrm{~mm}$ \\
\hline Detector Intrinsic Resolution & $R_{i}$ & $800 \mu \mathrm{m}$ \\
\hline PS-PMT size & $H$ & $50 \times 50 \mathrm{~mm}^{2}$ \\
\hline \hline
\end{tabular}

an area equivalent polygon. Using these $49 \mathrm{~mm}$ detectors in a polygonal configuration we are limited to a discrete number of detector distances $L: 18.2 \mathrm{~mm}, 27.6 \mathrm{~mm}, 36.3 \mathrm{~mm}$, $44.6 \mathrm{~mm}, 52.7 \mathrm{~mm}, 60.7 \mathrm{~mm}, 68.7 \mathrm{~mm}$, for a triangular, square, pentagonal, hexagonal, heptagonal, octagonal, nonagonal configuration, respectively. Table 1 summarizes the final design constraints.

\section{B. System optimization}

95 
of the field-of-view can be calculated as:

$$
\begin{aligned}
S & =n_{t x} n_{a x} \frac{a^{2}}{16 l^{2}} \cos ^{3} \alpha \\
& =\frac{2 \pi H \sqrt{1-\frac{d_{t x}^{2}}{4 l^{2}}} \frac{R_{g}^{2}}{16 L} f\left(\alpha_{\max }\right),}{d_{a x}^{2}}
\end{aligned}
$$

110

with $l$ the pinhole distance, $L$ the detector distance, $n_{a x}$ and $n_{t x}$ the axial and transaxial number of pinholes, $R_{g}$ the collimator geometric resolution and $a$ the pinhole aperture diameter. $f\left(\alpha_{\max }\right)$ is a correction factor for the sensitivity decrease due to oblique incidence angle $\alpha$ of axially off-center pinholes. In a cylindrical detector arrangement, in contrast to a spherical detector arrangement, L appears in the denominator of the sensitivity equation (equation 1). Therefore, the optimal sensitivity will be reached at realistic system dimensions. This can be seen in figure 3 (a), where we plotted the sensitivity as a function of collimator distance $l$, for a number of fixed detector distances $L$. It is clear from this plot that the optimal pinhole distance should be smaller than our pre-imposed value of $20 \mathrm{~mm}$. The optimal values of $l$ are reached at lower values, respectively at 12, 14 and $15 \mathrm{~mm}$ for detector distances $L$ of 35,40 or $45 \mathrm{~mm}$. With respect to the constraint that a mouse or rat head should be easily accommodated inside the scanner, we fix $l=l_{\min }$ at $20 \mathrm{~mm}$. For a fixed pinhole distance $l$ of $20 \mathrm{~mm}$, figure 3 (b) plots $S$ as a function of $L$ for different values of the intrinsic spatial resolution $R_{i}$. The intrinsic resolution enters in the sensitivity formula (equation 1) through the collimator resolution $R_{g}$ that is varied by changing the pinhole aperture size (cfr. figure 2).

From the considerations in section II A 2 with respect to the rectangular detectors, we best choose a pentagonal or a hexagonal configuration since both lie closest to the maximum sensitivity. From figure 3 (c) and (d), we see that a pentagonal arrangement has lower peak sensitivity than a hexagon. However, the pentagonal system will have more pinholes looking at the FOV which will enhance sampling completeness within the FOV. The hexagonal detector arrangement will have 62 pinholes simultaneously looking at the FOV while the pentagonal system will have 115 pinholes looking at the FOV. For the constraints of table 1, sensitivity would quickly decrease for a lower number of detectors, while the use of seven detectors or more would result in a number of pinholes that is inadequate for sampling completeness in a stationary system. 
TABLE II. Optimal and simulated parameters for a pentagonal and hexagonal system

\begin{tabular}{clllll}
\hline & \multicolumn{2}{c}{ Optimal } & & \multicolumn{2}{c}{ Simulated } \\
\cline { 2 - 3 } \cline { 5 - 6 } & Pentagon Hexagon & & Pentagon Hexagon \\
\hline \hline number of pinholes & & 115 & 62 & 100 & 54 \\
magnification $m$ & 0.81 & 1.23 & 0.88 & 1.23 \\
$l(m m)$ & 20 & 20 & 17.9 & 19.0 \\
$L(m m)$ & 36.3 & 44.6 & 33.7 & 42.4 \\
$a(\mu m)$ & $393 \mu m$ & $627 \mu m$ & $393 \mu m$ & $627 \mu m$ \\
$S_{\text {opt }}$ & $0.32 \%$ & $0.41 \%$ & $\mathrm{~N} / \mathrm{A}$ & $\mathrm{N} / \mathrm{A}$ \\
$R_{g}(\mathrm{~mm})$ & 0.980 & 1.23 & $\mathrm{~N} / \mathrm{A}$ & $\mathrm{N} / \mathrm{A}$ \\
\hline \hline
\end{tabular}

\section{Simulations}

\section{System configurations}

The outcome of the optimization for both geometries is summarized in table II. Although these values are optimal, they are not practical to be used. It is for instance not practical to distribute 115 pinholes over 5 detectors, or to divide 62 pinholes over 6 detectors. Furthermore, both detector and collimator transaxial geometries were polygonal as shown in figure 4. The polygon dimensions were normalized for detector surface to their ideal circular counterparts. The final system parameters that were used for simulation are summarized in table II. Since we based ourselves on a FOV of $12 \mathrm{~mm}$, we are either limited to small activity distributions or we have to scan the animal around as is done in the U-SPECT system [17]. Another option, proposed by Funk [10] is to let every pinhole look at a slightly different portion of the FOV. This allows the subject to remain completely stationary if only a small axial portion has to be scanned. We calculated the volume sensitivity and the number of pinholes seen by our simulated systems. 
A simulation and reconstruction platform, previously developed in our research group [24, 25], was used to simulate the multi-pinhole systems described in the previous sections. It consists of a ray-tracing algorithm, known as Joseph's method [26], for efficiently calculating the system matrix elements. System resolution modeling was performed by tracing multiple (456) rays through the pinhole aperture. Detector intrinsic resolution was modeled using a space-invariant Gaussian blurring kernel. Resolution and sensitivity effective pinhole apertures were taken into account and sensitivity is calculated according to [27] and [28] to model the effect of photon penetration. Sensitivity was taken into account in the pixel driven forward projector by:

$$
S=\frac{a_{s e}^{2}}{16 f^{2}} \cos \alpha
$$

with $f=(L-l)$ the focal length, $a_{\text {se }}$ the sensitivity effective aperture and $\alpha$ the incidence angle. Since we used a pixel driven projector, the number of rays passing through a voxel at distance 1 from the pinhole will be inversely proportional to the square of this distance. This means that there is already an inherent correction for the $1 / l^{2}$ sensitivity effect. Furthermore, we compensate for the fact that the pixel driven sampling would put a higher flux through a certain voxel when the detector is further away from the pinhole, by dividing the sensitivity by $f^{2}$. Scatter and attenuation were not modeled.

\section{Sampling completeness}

Axial sampling was investigated with a Defrise phantom, which consists of alternating hot and cold disks (each $24 \mathrm{~mm}$ diameter, $1 \mathrm{~mm}$ thickness). This phantom was simulated using a $256 \times 256 \times 256$ matrix with $200 \mu \mathrm{m}$ isotropic voxels. No noise was added to the data. Image reconstruction was performed using 500 Maximum Likelihood Expectation Maximization (MLEM) iterations to a $128 \times 128 \times 128$ voxel grid. Voxel size in the reconstructed image was $300 \mu \mathrm{m}$. 


\section{Reconstructed resolution}

Reconstructed resolution was evaluated using a hot rod phantom of $28 \mathrm{~mm}$ diameter and $18 \mathrm{~mm}$ height. Six hot rod segments with respective diameters of $700 \mu \mathrm{m}, 800 \mu \mathrm{m}, 900 \mu \mathrm{m}$, $1.0 \mathrm{~mm}, 1.2 \mathrm{~mm}$ and $1.4 \mathrm{~mm}$ were simulated using a $256 \times 256 \times 256$ matrix with $200 \mu \mathrm{m}$ hot-rod-to-background contrast. No noise was added to the data. Image reconstruction was performed using 500 Maximum Likelihood Expectation Maximization (MLEM) iterations to a $128 \times 128 \times 128$ voxel grid. Voxel size in the reconstructed image was $300 \mu \mathrm{m}$.

\section{MOBY mouse phantom}

185

To evaluate the system performance in a realistic imaging situation, a MOBY mouse phantom [29], with organ activity according to Tc-99m-tetrofosmin tracer biodistribution 3 hours post-injection [30], was simulated. No heart beat or breathing was simulated and only one time frame was used for generating the phantom. The activity values for the gallbladder, which has high tracer uptake and is located near the heart, were obtained from a recent study by Branderhorst et al. [31]. Additionally, to make the task even more challenging, we increased liver activity concentration to the heart uptake concentration. Simulated organ activities can be found in table III. The axial extent of the MOBY phantom was $37.12 \mathrm{~mm}$ and the phantom was simulated using a $256 \times 256 \times 256$ matrix with $145 \mu \mathrm{m}$ isotropic voxels. This is the standard voxel size when the phantom is generated at a $256^{3}$ grid. Poisson noise was added to the projection data taking into account the sensitivity in each voxel of the phantom, assuming a scan time of 45 minutes and an injection of $50 \mathrm{MBq}$. 500 MLEM iterations were used for reconstruction. Inter-update-smoothing was performed with an empirically determined Gaussian kernel $(\sigma=0.38 \mathrm{~mm})$. The reconstructed voxel size was the same as for the Defrise and hot rod phantoms $(300 \mu \mathrm{m})$. 
TABLE III. Simulated organ activities relative to the heart

wall activity

\begin{tabular}{ll}
\hline Organ & Relative value \\
\hline \hline Heart & 1.00 \\
Liver & 1.00 \\
Gallbladder & 7.63 \\
Intestines & 2.26 \\
Lung & 0.11 \\
Stomach & 0.94 \\
Kidney & 0.71 \\
Background & 0.16 \\
\hline
\end{tabular}

\section{RESULTS}

\section{A. Volume sensitivity, angular sampling and total FOV}

After optimizing the system where we assumed truncated pinhole projections (only $12 \mathrm{~mm}$ transverse FOV for each pinhole), we let every pinhole look at a different location, resulting through a map with the number of pinholes that see each voxel are shown, respectively 
for the pentagonal and hexagonal system. In figure 6 (c), one dimensional profiles for $\mathrm{y}=0$ are drawn. We see that 70 pinholes and 35 pinholes look at the central portion of the transverse FOV, decreasing to 25 and 20 pinholes as we move to the edge of the FOV, for the pentagonal and hexagonal system respectively. The average number of pinholes seen is 38 and 23 , respectively.

\section{B. Defrise reconstructions}

The reconstructed images of the micro Defrise phantom after 500 MLEM iterations are shown in figure 7. Figures 7 (a) and (b) show a transverse and axial cross section through the originally simulated image together with the target FOV. Figures 7 (c) and (d) show the results for the pentagonal system while figures 7 (e) and (f) show reconstructions for the hexagonal system. The different hot disks of the Defrise phantom within the FOV can clearly be observed, even though there is activity extending outside the axial FOV. These images confirm that axial sampling for both systems is good. Notice that the sampling in the hexagonal system is slightly worse compared to the pentagonal system.

\section{Hot rod image}

Figure 8 shows the reconstructed images of the hot rod phantom after 500 MLEM iterations. Figures 8 (a) and (b) show a transverse and axial cross section through the originally simulated phantom with indication of the rod diameters in each segment and the target FOV. Figures 8 (c) and (d) show the results for the pentagonal system while figures 8 (e) and (f) show reconstructions for the hexagonal system. The smallest rods (700 $\mu$ m diameter) are visible only on the pentagonal structure while the $800 \mu \mathrm{m}$ rods can be imaged with the hexagonal system. However, for the hexagonal system, the $800 \mu \mathrm{m}$ rods are not all very well defined, while this effect can only be observed near the edge of the FOV for the pentagonal system. 


\section{MOBY reconstructions}

The count level of the MOBY projections is 23.4 million and 26.1 million respectively for the pentagon and hexagon. Figure 9 shows the reconstructed images of the MOBY phantom after 500 MLEM iterations. Figures 9 (a) and (b) show a transverse and coronal cross section through the heart, respectively. Figures 9 (c) and (d) show the results for the pentagonal system while figures 9 (e) and (f) show reconstructions for the hexagonal system. Visually, the images look similar, with a slightly better definition of the left ventricular heart wall for the pentagonal structure. Also, the right ventricular wall is slightly better visible with the pentagonal structure.

\section{DISCUSSION}

Here, we proposed a stationary and small microSPECT design based on compact scintillation detectors with good intrinsic spatial resolution. Compact microSPECT systems are an attractive alternative for current state-of-the-art microSPECT systems that are not mobile and require a large physical space. Advantages are lower footprint of the system, lower overal cost and potential for increasing the performance as the detector technology evolves towards better intrinsic resolution. With the pentagonal system, we observed a reconstructed spatial resolution of around $0.7 \mathrm{~mm}$, which is in the range of the three commercial systems that we compared in [14]. These state-of-the-art systems have reconstructed spatial resolutions in the range of 0.58 to $0.76 \mathrm{~mm}$. Theoretical peak sensitivity of our pentagonal system is $0.32 \%$, which falls in the better end of the spectrum of sensitivities observed in the commercial systems, where sensitivities ranged from $0.06 \%$ to $0.4 \%$.

The pentagonal system has almost two times more pinholes compared to the hexagonal design. Therefore, it is expected to result in a better angular sampling of the radioactive tracer distribution inside the FOV. On the other hand, the hexagonal system has higher sensitivity and is expected to result in less noisy images. Axial sampling completeness, as verified with a Defrise disk phantom that almost covers the entire transverse FOV, was proven good by visual inspection of the reconstructed images. Slightly better sampling is observed with the pentagonal system. The reconstructed resolution was investigated using a hot rod phantom with rod diameters ranging from $700 \mu \mathrm{m}-1.4 \mathrm{~mm}$. The pentagonal struc- 
ture reveals a reconstructed resolution of $700 \mu \mathrm{m}$ while the hexagonal system has slightly worse spatial resolution, namely $800 \mu \mathrm{m}$. Since the target resolution was similar for both systems, theoretically, the reconstructed spatial resolution on both systems should be the same. However, in a reconstructed image, angular sampling might play a role in accurately

\section{CONCLUSION}

To determine the optimal configuration of our high space-bandwidth scintillation detectors, this study used a theoretical optimization method [23]. The resulting 'optimal' systems were simulated and good sampling was demonstrated. It was shown that a reconstructed spatial resolution of approximately $800 \mu \mathrm{m}$ can be achieved and that we can successfully reconstruct an image with realistic noise levels in a pre-clinical context. Such a system can be useful for dynamic SPECT imaging. 


\section{ACKNOWLEDGMENTS}

This work is funded by the Research Foundation - Flanders (FWO, Belgium), by the

\section{REFERENCES}

[1] Franc L.F., Acton P.D., Mari C., Hasegawa B.H.. Small-Animal SPECT and SPECT/CT: Important Tools for Preclinical Investigation Journal of Nuclear Medicine. 2008;49:16511663.

[2] Beekman F., Have F.. The pinhole: gateway to ultra-high-resolution three-dimensional radionuclide imaging European Journal of Nuclear Medicine. 2007;34:151-161.

[3] McElroy D.P., MacDonald L.R., Beekman F.J., et al. Performance Evaluation of A-SPECT: A High Resolution Desktop Pinhole SPECT System for Imaging Small Animals IEEE Transactions on Nuclear Science. 2002;49:2139-2147.

[4] Schramm N.U., Ebel G., Engeland U., Schurrat T., Behe M., Behr T.M.. High-resolution SPECT using multipinhole collimation IEEE Transactions on Nuclear Science. 2003;50:315320 .

[5] Furenlid L.R., Wilson D.W., Pietraski P.J., Crawford M.J., Barrett H.H.. FastSPECT II: a second-generation high-resolution dynamic SPECT imager IEEE Transactions on Nuclear Science. 2004;51:631-635.

[6] Furenlid L.R., Moore J.W., Freed M., et al. Adaptive small-animal SPECT/CT. IEEE 2008 International Symposium on Biomedical Imaging 2008.

[7] Lackas C., Schramm N.U., Hoppin J.W., Engeland U., Wirrwar A., Halling H.. T-SPECT: A novel imaging technique for small animal research IEEE Transactions on Nuclear Science. 2005;52:181-187. 
[8] Beekman F.J., Have F., Vastenhouw B., et al. U-SPECT-I: A novel system for submillimeterresolution tomography with radiolabeled molecules in mice Journal of Nuclear Medicine. $2005 ; 46: 1194-1200$.

[9] Kim H., Furenlid L.R., Crawford M.J., et al. SemiSPECT: a small-animal single-photon emission computed tomography (SPECT) imager based on eight cadmium zinc telluride (CZT) detector arrays Medical Physics. 2006;33:465-474.

[10] Funk T., Després P., Barber W.C., Shah K.S.., Hasegawa B.H.. A multipinhole small animal SPECT system with submillimeter spatial resolution Medical Physics. 2006b;33:1259-1268.

[11] Vunckx K., Beque D., Defrise M., Nuyts J.. Single and Multipinhole Collimator Design Evaluation Method for Small Animal SPECT IEEE Transactions on Medical Imaging. 2008;27.

[12] Mok G.S., Tsui B.M.W., Beekman F.J.. The effects of object activity distribution on multiplexing multi-pinhole SPECT Physics in Medicine and Biology. 2011;56:2635-2650.

[13] Have F., Vastenhouw B., Rentmeester M., Beekman F.J.. System calibration and statistical image reconstruction for ultra-high resolution stationary pinhole SPECT IEEE Transactions on Medical Imaging. 2008;27:960-71.

[14] Deleye S., Van Holen R., Verhaeghe J., Vandenberghe S, Stroobants S., Staelens S... Performance evaluation of small animal multipinhole uSPECT scanners for mouse imaging European Journal of Nuclear Medicine. 2013;40:744-758.

[15] Rogulski M.M., Barber H.B., Barrett H.H., Shoemaker R.L., Woolfenden J.M.. Ultra-highresolution brain SPECT imaging: simulation results IEEE Transactions on Nuclear Science. 1993;40:1123-1129.

[16] Goorden M.C., Rentmeester M.C.M, Beekman F.J.. Theoretical analysis of full-ring multipinhole brain SPECT Physics in Medicine and Biology. 2009;54:6593-6610.

[17] Have F., Vastenhouw B., Ramakers R.M., et al. U-SPECT-II: an ultra-high-resolution device for molecular small-animal imaging. Journal of Nuclear Medicine. 2009;50:599-605.

[18] Lin J.Y., Meikle S.R.. SPECT using asymmetric pinholes with truncated projections Physics in Medicine and Biology. 2011;56:4103-4118.

[19] Deprez K., Van Holen R., Staelens S., Vandenberghe S.. Design of a high resolution scintillator based SPECT detector (SPECTatress) Nuclear Instruments and Methods A. 2010;648:107110. 
[20] Deprez K., Vandenberghe S., Vandeghinste B., Van Holen R.. FlexiSPECT: A SPECT System Consisting of a Compact High-Resolution Scintillation Detector (SPECTatress) and a Lofthole Collimator IEEE Transactions on Nuclear Science. 2013;60:53-64.

[21] Barrett H.H., Hunter W.C.J., Miller B.W., Moore S.K., Chen Y., Furenlid L.R.. A High Resolution Scintillator Based SPECT Detector with Digital Pulse Processing (SPECTatress) IEEE Transactions on Nuclear Science. 2009;56:725-735.

[22] Hesterman J.Y., Caucci L., Kupinski M.A., Barrett H.H., Furenlid L.R.. Maximum-Likelihood Estimation With a Contracting-Grid Search Algorithm IEEE Transactions on Nuclear Science. 2010;57:1077-1084.

[23] Nillius P., Danielsson M.. Theoretical Bounds and System Design for Multipinhole SPECT IEEE Transactions on Medical Imaging. 2010;29:1390-1400.

[24] Van Holen R., Moore J.W., Clarkson E.W., Furenlid L.R., Barrett H.H.. Design and Validation of an Adaptive SPECT System: AdaptiSPECT 2010 IEEE Nuclear Science Symposium and Medical Imaging Conference Record. 2010:2539-2544.

[25] Vandeghinste B., Vanhove C., De Beenhouwer J., Van Holen R., Vandenberghe S., Staelens S.. Absolute quantification in multi-pinhole micro-SPECT for different isotopes 2011 IEEE Nuclear Science Symposium and Medical Imaging Conference Record. 2011:3720-3724.

[26] Joseph P.M.. An Improved Algorithm for Reprojecting Rays Through Pixel Images IEEE Transactions on Medical Imaging. 1982;1:192-196.

[27] Metzler S.D., Bowsher J.E., Smith M.F., Jaszczak R.J.. Analytic determination of pinhole collimator sensitivity with penetration IEEE Transactions on Medical Imaging. 2010;20:730741.

[28] Accorsi R., Metzler S.D.. Analytic Determination of the Resolution-Equivalent Effective Diameter of a Pinhole Collimator IEEE Transactions on Medical Imaging. 2004;23:750-763.

[29] Segars W.P., Tsui B.M.W., Frey E.C., Johnson G.A., Berr S.S.. Development of a 4-D digital mouse phantom for molecular imaging research Molecular Imaging and Biology. 2004;6:149159.

[30] Amano S., Inoue T., Tomiyoshi K., Ando T., Endo K.. In vivo comparison of PET and SPECT radiopharmaceuticals in detecting breast cancer Journal of Nuclear Medicine. 1998;39:14241427. 
[31] Branderhorst W., Have F., Vastenhouw B., Viergever M.A., Beekman F.J.. Murine cardiac images obtained with focusing pinhole SPECT are barely influenced by extra-cardiac activity Physics in Medicine and Biology. 2012;57:717-732. 


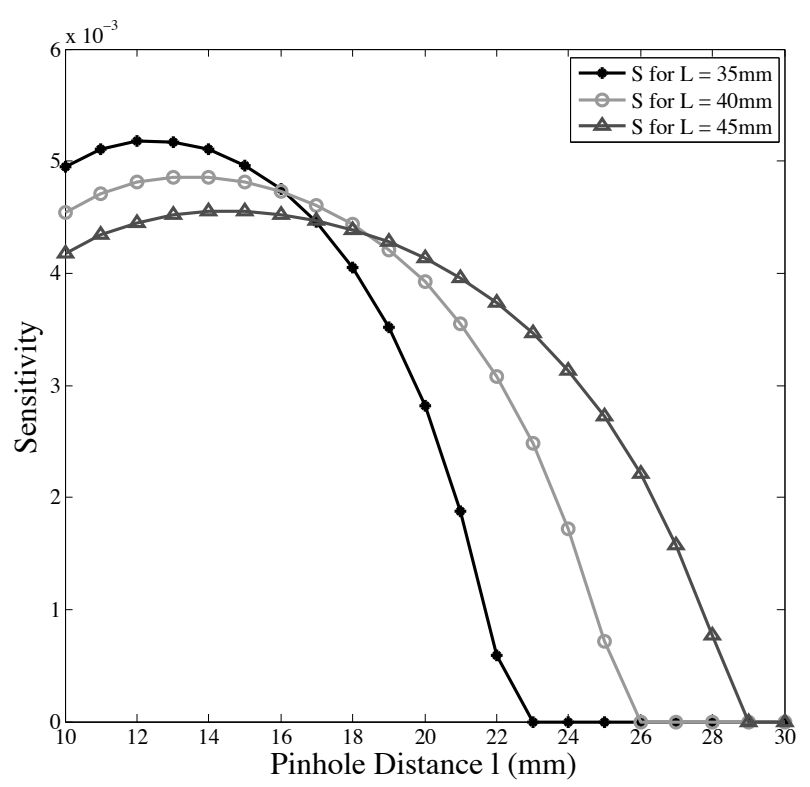

(a)



(c)

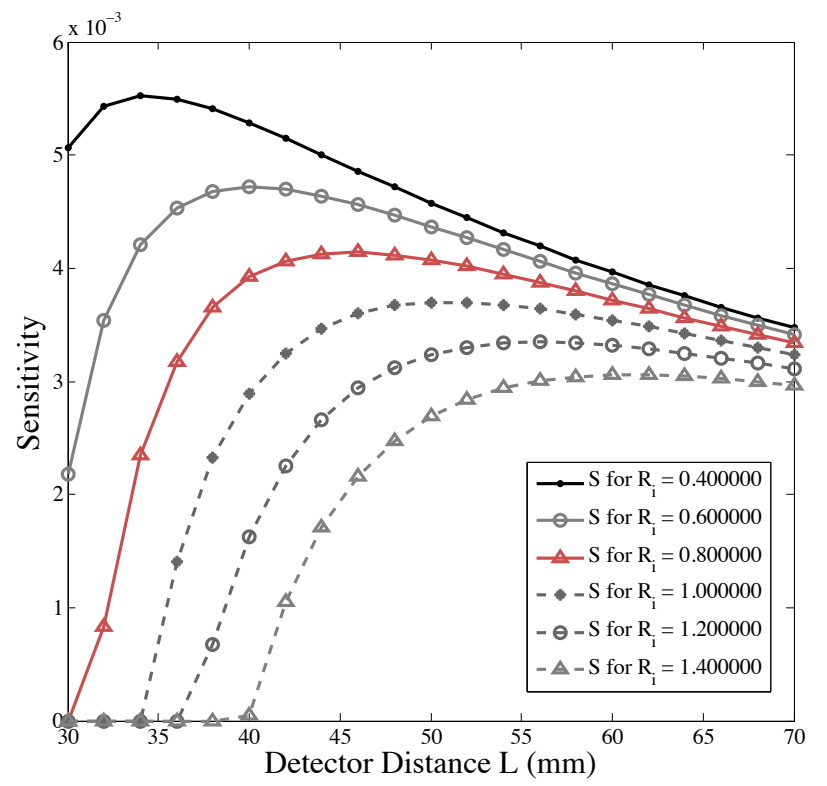

(b)

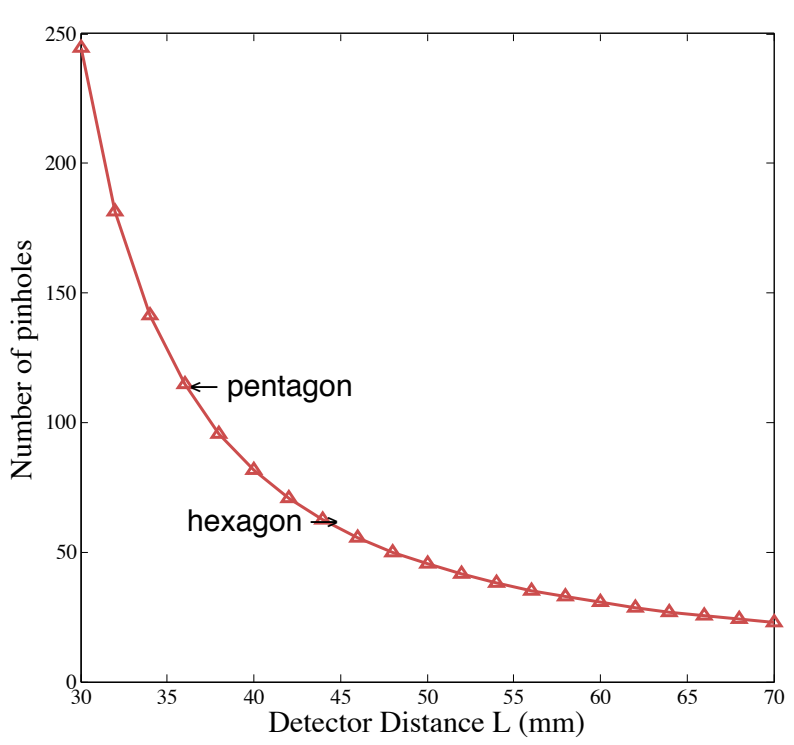

(d)

FIG. 3. In (a), the sensitivity is plotted as a function of pinhole distance $l$ for three different detector distances $L$. In (b), the sensitivity is plotted as a function of $L$, for $l=20 \mathrm{~mm}$ and for different values of intrinsic resolution $R_{i}$ while (c) shows (b) for $R_{i}=800 \mu \mathrm{m}$, equal to our intrinsic resolution. We also show the detector distances that agree with a pentagonal and a hexagonal detector arrangement. In (d), the number of pinholes is plotted as a function of detector distance $L$ with indication of the number of pinholes for a pentagon (115) and for a hexagon (62). 


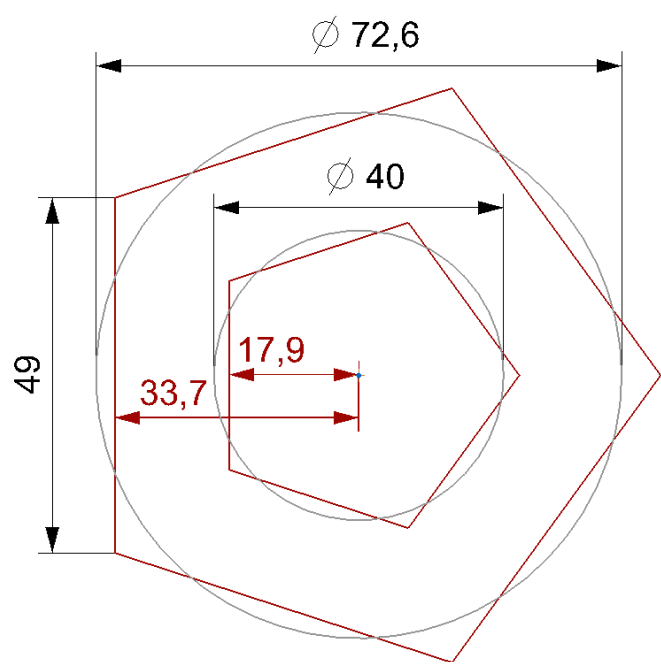

(a)



(c)

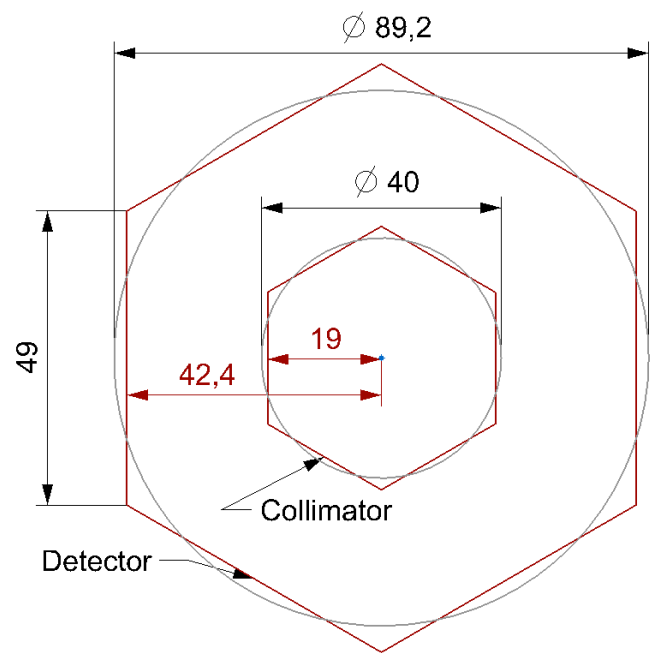

(b)

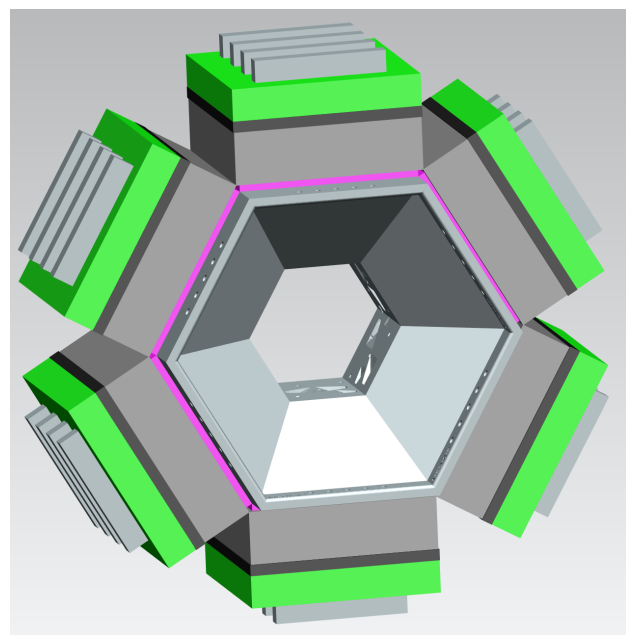

(d)

FIG. 4. The simulated SPECT system geometry definition. In (a) and (b), the pentagonal and hexagonal arrangements are shown, respectively. Polygon dimensions are area normalized to the ideal circular counterparts (dimensions are in $\mathrm{mm}$ ). In (c) and (d), a rendering of the respective simulated systems is shown. Centrally, the multipinhole collimators are shown, followed by the scintillator, PMTs and electronics. 


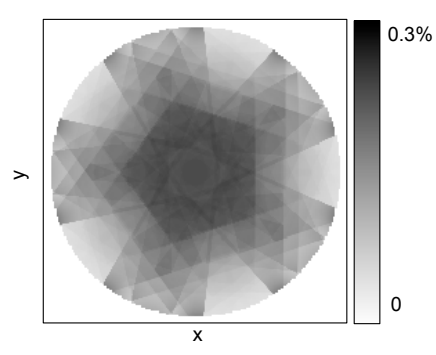

(a)

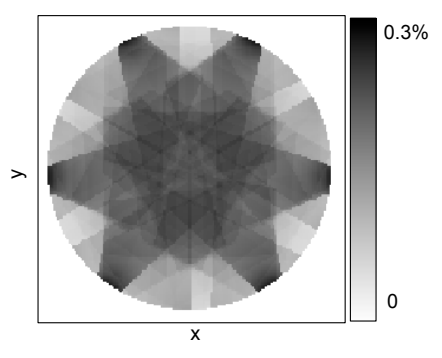

(b)

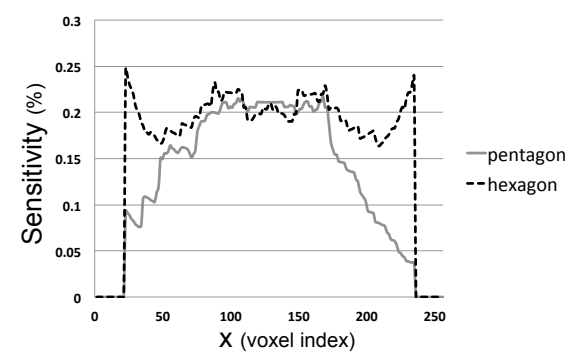

(c)

FIG. 5. Transverse sections through the sensitivity map (at $\mathrm{z}=0$ ) for (a) the pentagonal and (b) the hexagonal system for each pinhole looking at a different location in the FOV. In (c), a line profile through figures (a) and (b) is shown at $\mathrm{y}=0$.



(a)

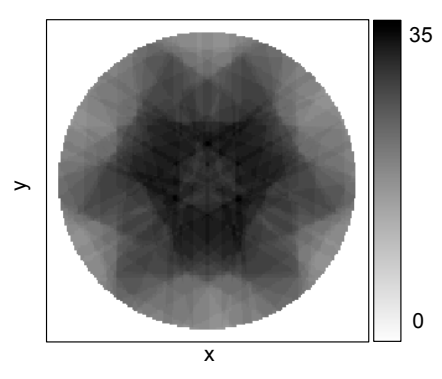

(b)

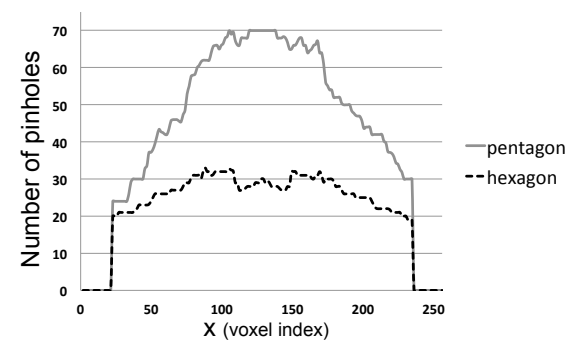

(c)

FIG. 6. Transverse sections through the map showing the number of pinholes seen in each voxel (at $\mathrm{z}=0$ ) for (a) the pentagonal and (b) the hexagonal system. In (c), a line profile through figures (a) and (b) is shown at $\mathrm{y}=0$. 


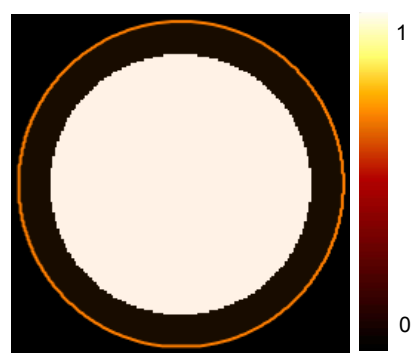

(a)

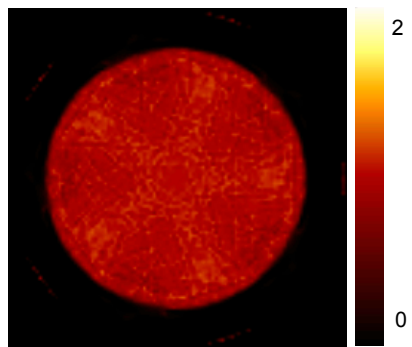

(c)

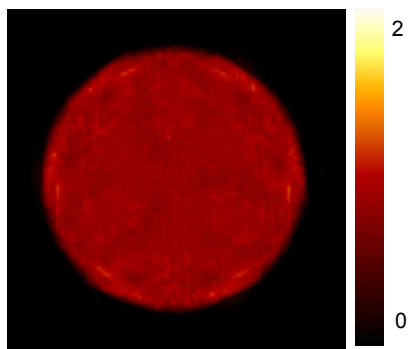

(e)

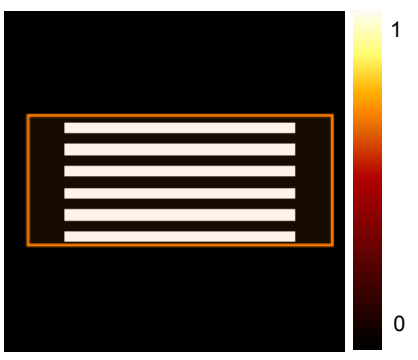

(b)

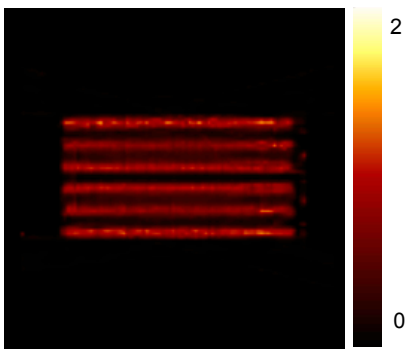

(d)

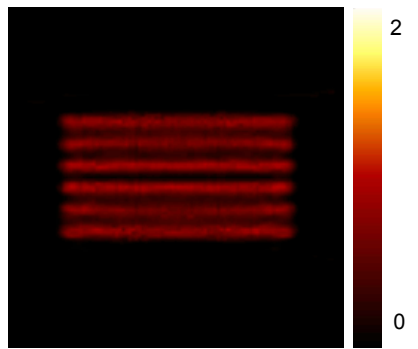

(f)

FIG. 7. (a) Transverse and (b) axial view of the Defrise phantom ( $\varnothing 24 \mathrm{~mm}$, height $18 \mathrm{~mm}$ ) and their respective reconstructions for $(\mathrm{c}),(\mathrm{d})$ the pentagonal and $(\mathrm{e}),(\mathrm{f})$ the hexagonal system. In figures (a) and (b), also the FOV ( $\varnothing 30 \mathrm{~mm}$, height $12 \mathrm{~mm}$ ) of the scanner is indicated in color. 


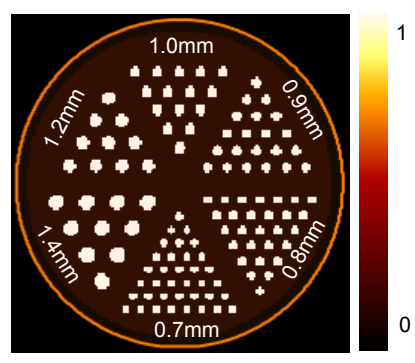

(a)

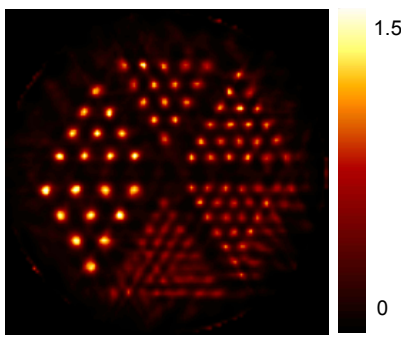

(c)



(e)

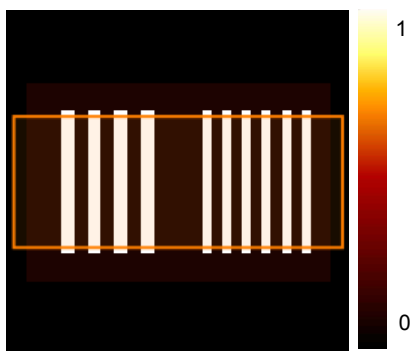

(b)

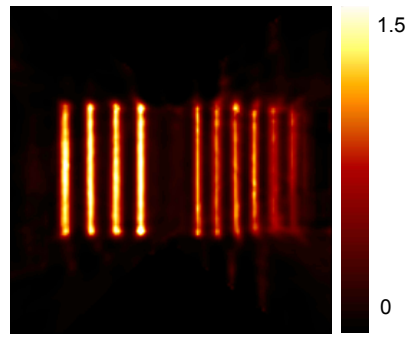

(d)



(f)

FIG. 8. (a) Transverse and (b) axial view of the hot rod phantom ( $\varnothing 28 \mathrm{~mm}$, height $18 \mathrm{~mm})$ and their respective reconstructions for $(\mathrm{c}),(\mathrm{d})$ the pentagonal and (e),(f) the hexagonal system. In figures (a) and (b), also the FOV ( $\varnothing 30 \mathrm{~mm}$, height $12 \mathrm{~mm})$ of the scanner is indicated in color. 


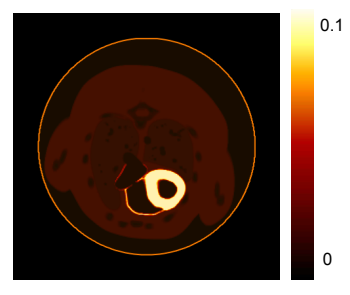

(a)

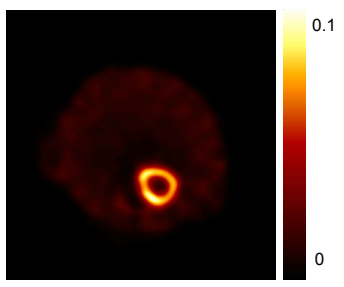

(c)

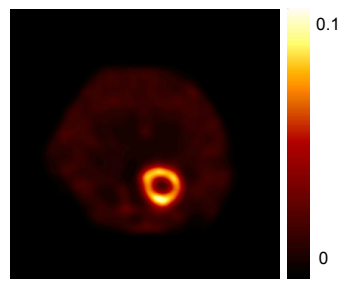

(e)

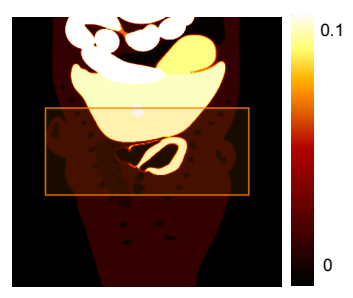

(b)

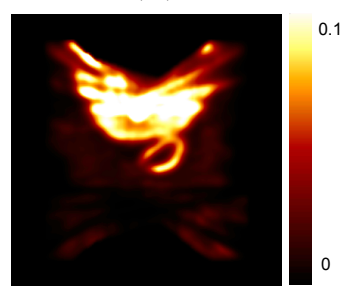

(d)

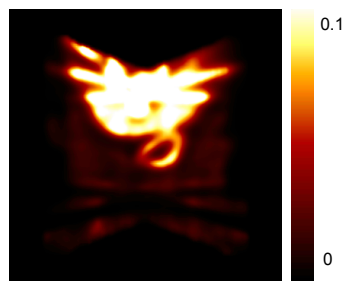

(f)

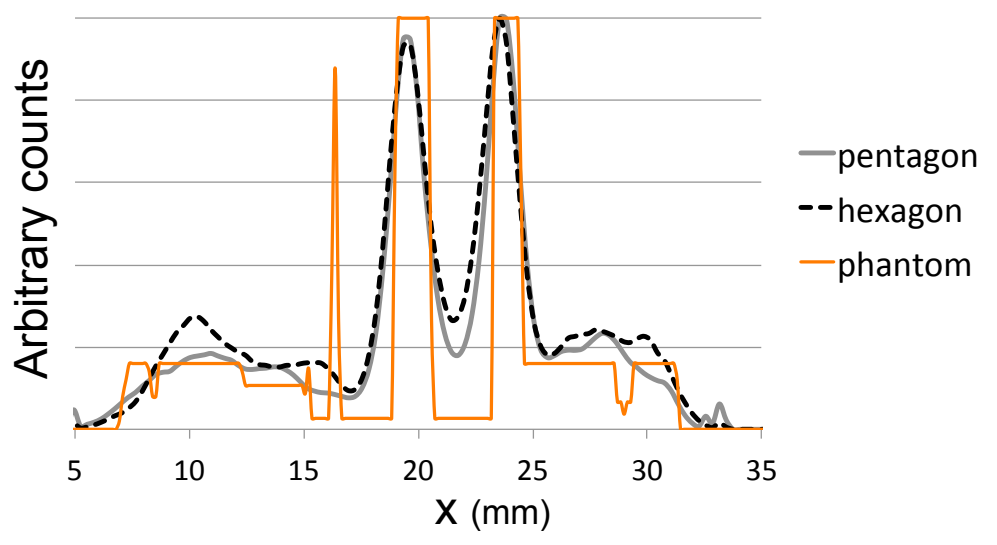

(g)

FIG. 9. Noisy reconstructions of the MOBY mouse phantom: (a) transverse and (b) coronal view through the heart of the MOBY phantom and their respective reconstructions for (c) and (d), the pentagonal and (e) and (f) the hexagonal system. In (g), a line profile through the coronal images of (b),(d) and (f) is shown. In figures (a) and (b), also the FOV ( $\varnothing 30 \mathrm{~mm}$, height $12 \mathrm{~mm}$ ) of the scanner is indicated in color (orange). 